PROCEEDINGS OF THE

AMERICAN MATHEMATICAL SOCIETY

Volume 130, Number 2, Pages 471-476

S 0002-9939(01)06053-1

Article electronically published on May 25, 2001

\title{
INNER DERIVATIONS AND NORM EQUALITY
}

\author{
MOHAMED BARRAA AND MOHAMED BOUMAZGOUR
}

(Communicated by Joseph A. Ball)

\begin{abstract}
We characterize when the norm of the sum of two bounded operators on a Hilbert space is equal to the sum of their norms.
\end{abstract}

\section{INTRODUCTION}

Let $H$ denote a complex Hilbert space and let $\mathcal{L}(\mathcal{H})$ denote the algebra of all bounded linear operators on $H$. For $A$ and $B$ in $\mathcal{L}(\mathcal{H})$, the equation

$$
\|A+B\|=\|A\|+\|B\|
$$

was studied by many authors; see for instance 1 , 8 and the references therein. In [1], it is shown that an operator $B$ satisfies the equation $\|I+B\|=1+\|B\|$ if and only if the norm $\|B\|$ lies in the approximate point spectrum of $B$; here $I$ denotes the operator identity on $H$. Another type of result in this direction (see Lin [8]) shows that if two operators $A$ and $B$ in $\mathcal{L}(\mathcal{H})$ satisfy equation $(*)$, then 0 must be in the approximate point spectrum of the operator $\|B\| A-\|A\| B$; moreover $\mathrm{C}$. L. Lin proved that the converse holds if either $A$ or $B$ is an isometric operator.

In section 2 of the present paper we shall give a necessary and sufficient condition for any pair of operators $A$ and $B$ in $\mathcal{L}(\mathcal{H})$ to satisfy equation $(*)$. Moreover, we show that in general the condition of $[8$ that 0 lies in the approximate point spectrum of $\|B\| A-\|A\| B$ is not necessary to get $\|A+B\|=\|A\|+\|B\|$.

In section 3 , we shall apply the main result of section 1 to the theory of inner derivations, and we thereby answer the question of L. Fialkow 3] concerning when the norm of the restriction of a derivation to a norm ideal in $\mathcal{L}(\mathcal{H})$ is independent of the ideal.

In order to state our results in detail, we first recall some notations and results from the literature. For $A \in \mathcal{L}(\mathcal{H})$, let $\sigma(A), \sigma_{a p}(A)$ and $r(A)$ denote respectively the spectrum, approximate point spectrum and spectral radius of $A$. Recall that a complex number $\lambda \in \sigma_{a p}(A)$ if there exists a unit sequence $\left\{x_{n}\right\}_{n} \subseteq H$ such that $\lim _{n}\left\|(A-\lambda) x_{n}\right\|=0$. Since the boundary of $\sigma(A)$ is contained in $\sigma_{a p}(A)$, then $\|A\| \in \sigma(A)$ if and only if $\|A\| \in \sigma_{a p}(A)$.

The numerical range of $A$ is defined by $W(A)=\{\langle A x, x\rangle: x \in H,\|x\|=1\}$ and the numerical radius of $A$ is defined by $w(A)=\sup \{|\lambda|: \lambda \in W(A)\}$. Note that

Received by the editors December 3, 1999 and, in revised form, June 27, 2000.

1991 Mathematics Subject Classification. Primary 47A10, 47A12, 47A30, 47B10, 47B20, 47B37, 47B47, 47D50.

Key words and phrases. Generalized derivation, norm, norm ideal, $S$-universal operator, numerical range, spectrum, quasi-nilpotent operator, hyponormal operator.

(C)2001 American Mathematical Society 
$\overline{W(A)}$ is a compact convex subset of the plane and $\sigma(A) \subseteq \overline{W(A)}[6]$, where the bar denotes the closure. From [6], it turns out that the norm $\|A\|$ lies in $\overline{W(A)}$ if and only if $\|A\|$ lies in $\sigma_{a p}(A)$.

\section{NORM EQUALITY}

In this section we shall characterize when the norm of the sum of two operators on $H$ is equal to the sum of their norms. The main result of this section is the following.

Theorem 2.1. Let $A, B \in \mathcal{L}(\mathcal{H})$ be non-zero. Then the equation $\|A+B\|=$ $\|A\|+\|B\|$ holds if and only if $\|A\|\|B\| \in \overline{W\left(A^{*} B\right)}$.

Proof. Suppose $\|A+B\|=\|A\|+\|B\|$. There exists a sequence of vectors $\left\{x_{n}\right\}_{n}$ with $\left\|x_{n}\right\|=1$ for each $n$ and such that $\lim _{n}\left\|A x_{n}+B x_{n}\right\|=\|A\|+\|B\|$. Since

$$
\left\|A x_{n}+B x_{n}\right\| \leq\left\|A x_{n}\right\|+\left\|B x_{n}\right\| \leq\|A\|+\left\|B x_{n}\right\| \leq\|A\|+\|B\|,
$$

then

$$
\lim _{n}\left(\|A\|+\left\|B x_{n}\right\|\right)=\|A\|+\|B\| .
$$

Hence we deduce that $\lim _{n}\left\|B x_{n}\right\|=\|B\|$. Similarly, we obtain $\lim _{n}\left\|A x_{n}\right\|=\|A\|$. From the identity

$$
\left\|A x_{n}+B x_{n}\right\|^{2}=\left\|A x_{n}\right\|^{2}+\left\|B x_{n}\right\|^{2}+2 \Re\left(\left\langle A^{*} B x_{n}, x_{n}\right\rangle\right) \quad(\Re: \text { real part }),
$$

we see that

$$
\lim _{n} \Re\left(\left\langle A^{*} B x_{n}, x_{n}\right\rangle\right)=\|A\|\|B\| .
$$

Since $\left|\left\langle A^{*} B x_{n}, x_{n}\right\rangle\right|=\left(\left(\Re\left(\left\langle A^{*} B x_{n}, x_{n}\right\rangle\right)\right)^{2}+\left(\Im\left(\left\langle A^{*} B x_{n}, x_{n}\right\rangle\right)\right)^{2}\right)^{\frac{1}{2}}$ (ऽ : imaginary part), and $\left|\left\langle A^{*} B x_{n}, x_{n}\right\rangle\right| \leq\left\|A^{*} B x_{n}\right\| \leq\|A\|\|B\|$, we infer that

$$
\lim _{n}\left|\left\langle A^{*} B x_{n}, x_{n}\right\rangle\right|=\|A\|\|B\| \text {. }
$$

Thus $\lim _{n} \Im\left(\left\langle A^{*} B x_{n}, x_{n}\right\rangle\right)=0$, whence $\lim _{n}\left\langle A^{*} B x_{n}, x_{n}\right\rangle=\|A\|\|B\|$, i.e., $\|A\|\|B\|$ $\in \overline{W\left(A^{*} B\right)}$.

For the converse, assume that $\|A\|\|B\| \in \overline{W\left(A^{*} B\right)}$. Pick a unit sequence $\left\{x_{n}\right\}_{n} \subseteq H$ such that $\lim _{n}\left\langle A^{*} B x_{n}, x_{n}\right\rangle=\|A\|\|B\|$. We know that $\left|\left\langle A^{*} B x_{n}, x_{n}\right\rangle\right| \leq$ $\left\|A x_{n}\right\|\|B\| \leq\|A\|\|B\|$, whence $\lim _{n}\left\|A x_{n}\right\|=\|A\|$. By a similar argument, we get $\lim _{n}\left\|B x_{n}\right\|=\|B\|$. Since $\left\|A x_{n}+B x_{n}\right\|^{2}=\left\|A x_{n}\right\|^{2}+\left\|B x_{n}\right\|^{2}+2 \Re\left(\left\langle A^{*} B x_{n}, x_{n}\right\rangle\right)$ and $\lim _{n} \Re\left(\left\langle A^{*} B x_{n}, x_{n}\right\rangle\right)=\|A\|\|B\|$, we deduce that $\lim _{n}\left\|A x_{n}+B x_{n}\right\|=\|A\|+$ $\|B\|$, whence $\|A+B\|=\|A\|+\|B\|$; the proof is complete.

Corollary 2.2. For two operators $A$ and $B \in \mathcal{L}(\mathcal{H})$, the following are equivalent:

1. There exists $\lambda \in \mathbb{C}$ with $|\lambda|=1$ such that $\|A+\lambda B\|=\|A\|+\|B\|$.

2. There exists $\lambda \in \mathbb{C}$ with $|\lambda|=1$ such that $\lambda\|A\|\|B\| \in \overline{W\left(B^{*} A\right)}$.

3. There exists $\lambda \in \mathbb{C}$ with $|\lambda|=1$ such that $\lambda\|A\|\|B\| \in \sigma_{a p}\left(B^{*} A\right)$.

4. $w\left(A^{*} B\right)=\left\|A^{*} B\right\|=\|A\|\|B\|$.

5. $r\left(A^{*} B\right)=\left\|A^{*} B\right\|=\|A\|\|B\|$.

Proof. The equivalence $1 \Leftrightarrow 2$ follows from Theorem 2.1. The implications $3 \Rightarrow$ $2 \Rightarrow 4$ and $5 \Rightarrow 3$ are obvious. The implication $4 \Rightarrow 5$ follows from the general fact: For any operator $T$ on $H, r(T)=\|T\|$ if and only if $w(T)=\|T\|$ see $([6])$. 
Corollary 2.3. Let $A, B \in \mathcal{L}(\mathcal{H})$ be non-zero. If $\|A\|\|B\| \in \overline{W\left(A^{*} B\right)}$, then $0 \in$ $\sigma_{a p}(\|B\| A-\|A\| B)$. The converse holds if any one of $A$ or $B$ is an isometric operator.

Proof. If $\|A\|\|B\| \in \overline{W\left(A^{*} B\right)}$, then one can find a sequence of vectors $\left\{x_{n}\right\}_{n}$ with $\left\|x_{n}\right\|=1$ for each $n$ such that $\lim _{n}\left\langle A^{*} B x_{n}, x_{n}\right\rangle=\|A\|\|B\|$. So $\lim _{n} \Re\left\langle A^{*} B x_{n}, x_{n}\right\rangle$ $=\|A\|\|B\|$. Since $\left|\left\langle A^{*} B x_{n}, x_{n}\right\rangle\right| \leq\left\|A x_{n}\right\|\left\|B x_{n}\right\| \leq\|A\|\left\|B x_{n}\right\| \leq\|A\|\|B\|$, then $\lim _{n}\left\|B x_{n}\right\|=\|B\|$ and similarly we get $\lim _{n}\left\|A x_{n}\right\|=\|A\|$. Now

\|\|$B\left\|A x_{n}-\right\| A\left\|B x_{n}\right\|^{2}=\|B\|^{2}\left\|A x_{n}\right\|^{2}+\|A\|^{2}\left\|B x_{n}\right\|^{2}-2\|A\|\|B\| \Re\left(\left\langle A^{*} B x_{n}, x_{n}\right\rangle\right)$,

so $\lim _{n}\|\| B\left\|A x_{n}-\right\| A\left\|B x_{n}\right\|=0$, that is, $0 \in \sigma_{a p}(\|B\| A-\|A\| B)$.

To prove the converse, suppose for instance that $B$ is an isometry. The condition $0 \in \sigma_{a p}(A-\|A\| B)$ implies the existence of unit sequence $\left\{x_{n}\right\}_{n} \subseteq H$ such that $\lim _{n}\left\|A x_{n}-\right\| A\left\|B x_{n}\right\|=0$. From the inequality $\left\|A x_{n}-\right\| A\left\|B x_{n}\right\| \geq\left|\left\|A x_{n}\right\|-\|A\|\right|$, we conclude that $\lim _{n}\left\|A x_{n}\right\|=\|A\|$. But $\lim _{n}\left\langle\left(A x_{n}-\|A\| B x_{n}\right), A x_{n}\right\rangle=0$. Hence we infer that

$$
\lim _{n}\left\langle A^{*} B x_{n}, x_{n}\right\rangle=\|A\|,
$$

whence

$$
\|A\| \in \overline{W\left(A^{*} B\right)}
$$

which proves the corollary.

Remark 2.4. 1) Corollary 2.3 may be established by using Theorem 2.1 above and Theorem 1 of $[8]$.

2) In [8], it is proved that if $A, B \in \mathcal{L}(\mathcal{H})$, then $0 \in \sigma_{a p}(\|B\| A-\|A\| B)$. The following example shows that in general the condition $0 \in \sigma_{a p}(\|B\| A-\|A\| B)$ does not imply equation $(*)$.

Example 2.5. Let

$$
A=\left(\begin{array}{ll}
1 & 0 \\
1 & 0
\end{array}\right) \text { and } B=\left(\begin{array}{ll}
0 & -1 \\
0 & -1
\end{array}\right)
$$

on $H=\mathbb{C}^{2}$. Since

$$
A^{*} A=\left(\begin{array}{cc}
2 & 0 \\
0 & 0
\end{array}\right) \text { and } B^{*} B=\left(\begin{array}{cc}
0 & 0 \\
0 & 2
\end{array}\right)
$$

then

$$
\|A\|=\|B\|=\sqrt{2} .
$$

Next

$$
A-B=\left(\begin{array}{ll}
1 & 1 \\
1 & 1
\end{array}\right),
$$

so $\sigma_{a p}(A-B)=\{0,2\}$, but $\|A-B\|=2<2 \sqrt{2}=\|A\|+\|B\|$. 


\section{Applichtion to $S$-Universal operators}

Before stating the main result of this section we need some additional preliminaries. For a bounded operator $A \in \mathcal{L}(\mathcal{H})$, we mean by $L_{A}$ (respectively $R_{A}$ ) the left (respectively right) multiplication by $A$ on $\mathcal{L}(\mathcal{H})$, and $\delta_{A}$ denotes the inner derivation $L_{A}-R_{A}$.

Let $\left(J,\|\cdot\|_{J}\right)$ be a norm ideal in the sense of [5]. If $X \in J$ and $A, B \in \mathcal{L}(\mathcal{H})$, then $\|A X B\|_{J} \leq\|A\|\|X\|_{J}\|B\|$, with equality if $A$ and $B$ are unitary. Let $\mathcal{C}_{p}(\mathcal{H})$ denote the Schatten $p$-ideal, $1 \leq p \leq \infty$; see for instance [5] or [9]. The space $\mathcal{C}_{p}(\mathcal{H})$ consists of the compact operators $X$ such that $\sum_{j} S_{j}^{p}(X)<\infty$, where $\left\{S_{j}(X)\right\}_{j}$ denotes the sequence of the singular values of $X$. For $X \in \mathcal{C}_{p}(\mathcal{H}) \quad(1 \leq p \leq \infty)$, we set $\|X\|_{P}=\left(\sum_{j} S_{j}^{p}(X)\right)^{1 / p}$, where, by convention, $\|X\|_{\infty}=S_{1}(X)$ is the usual operator norm of $X$. Then $\left(\mathcal{C}_{p}(\mathcal{H}),\|\cdot\|_{p}\right)$ is a norm ideal. Moreover, $\left(\mathcal{C}_{p}(\mathcal{H}) 2,\|\cdot\|_{2}\right)$ is a Hilbert space with inner product defined by

$$
\langle X, Y\rangle=\operatorname{tr}\left(Y^{*} X\right) \quad\left(X, Y \in \mathcal{C}_{2}(\mathcal{H})\right),
$$

where $t r$ denotes the usual trace functional.

Let $\left(J,\|.\|_{J}\right)$ be a norm ideal in $\mathcal{L}(\mathcal{H})$ and let $A \in \mathcal{L}(\mathcal{H})$. If $X \in J$, then $\|A X-X A\|_{J}=\|(A-\lambda) X-X(A-\lambda)\|_{J} \leq 2\|A-\lambda\|\|X\|_{J}$ for all $\lambda \in \mathbb{C}$. Hence $\|A X-X A\|_{J} \leq 2 \inf _{\lambda \in \mathbb{C}}\|A-\lambda\|\|X\|_{J}$. Since $\left\|\delta_{A}\right\|=2 \inf _{\lambda \in \mathbb{C}}\|A-\lambda\|$ (see [11]), we conclude that $\|A X-X A\|_{J} \leq\left\|\delta_{A}\right\|\|X\|_{J}$. Thus the restriction $\delta_{J, A}$ of $\delta_{A}$ to $J$ defines a bounded linear operator on $\left(J,\|\cdot\|_{J}\right)$ and $\left\|\delta_{J, A}\right\| \leq\left\|\delta_{A}\right\|$ for each norm ideal $J$ in $\mathcal{L}(\mathcal{H})$. For simplicity of notation, we write $\delta_{2, A}$ instead of $\delta_{\mathcal{C}_{2}(\mathcal{H}), A}$.

The numerical range of an inner derivation on norm ideals in $\mathcal{L}(\mathcal{H})$ was studied by several authors; see for instance [7] or [10]. In [10] S. Shaw considered inner derivations $\delta_{S, A}$ acting on subspaces $\left(S,\|\cdot\|_{S}\right)$ of $\mathcal{L}(\mathcal{E})(E$ : Banach space) which satisfy axioms like those of norm ideals. In particular, he proved that $\overline{W\left(\delta_{2, A}\right)}=$ $\overline{W(A)}-\overline{W(A)}$.

Let $K$ be a non-empty bounded subset of the plane. The diameter of $K$ is defined by $\operatorname{diam}(K)=\sup _{\alpha, \beta \in K}|\alpha-\beta|$. For $A$ in $\mathcal{L}(\mathcal{H})$, we see from above that $w\left(\delta_{2, A}\right)=$ $\operatorname{diam}(W(A))$. On the other hand, it turns out [3] that $\sigma\left(\delta_{2, A}\right)=\sigma(A)-\sigma(A)$. Hence we deduce that $r\left(\delta_{2, A}\right)=\operatorname{diam}(\sigma(A))$.

Definition 3.1. An operator $A \in \mathcal{L}(\mathcal{H})$ is $S$-universal if $\left\|\delta_{J, A}\right\|=2 \inf \{\|A-\lambda I\|$ : $\lambda \in \mathbb{C}\}$ for each norm ideal $J$.

The concept of an $S$-universal operator was introduced by L. Fialkow 3], who studied criteria for $S$-universality and posed several questions in this context. In particular, it is proved in 3 . that $\operatorname{diam}(W(A)) \leq\left\|\delta_{J, A}\right\| \leq 2 \inf _{\lambda \in \mathbb{C}}\|A-\lambda\|$ for each operator $A \in \mathcal{L}(\mathcal{H})$ and each norm ideal $J$. The main result of this section is the following theorem.

Theorem 3.2. Let $A \in \mathcal{L}(\mathcal{H})$ be non-zero. Then $\left\|\delta_{2, A}\right\|=\left\|\delta_{A}\right\|$ if and only if $r\left(\delta_{2, A}\right)=\left\|\delta_{A}\right\|$.

Proof. Assume that $\left\|\delta_{2, A}\right\|=\left\|\delta_{A}\right\|$. By Theorem 8 of 11, we have $\left\|\delta_{A}\right\|=$ $2 \inf _{\lambda \in \mathbb{C}}\|A-\lambda\|$. By a compactness argument, there exists $\mu \in \mathbb{C}$ such that $\inf _{\lambda \in \mathbb{C}}\|A-\lambda\|=\|A-\mu\|$. Hence $\left\|\delta_{2, A}\right\|=2\|A-\mu\|$. Since $\delta_{2, A}=\delta_{2, A-\mu}=$ $L_{2, A-\mu}-R_{2, A-\mu}$, it follows that

$$
\left\|L_{2, A-\mu}-R_{2, A-\mu}\right\|=2\|A-\mu\| .
$$


On the other hand, $\left\|L_{2, A-\mu}\right\|=\|A-\mu\|$ and $\left\|R_{2, A-\mu}\right\|=\|A-\mu\|$. Hence

$$
\left\|L_{2, A-\mu}-R_{2, A-\mu}\right\|=\left\|L_{2, A-\mu}\right\|+\left\|R_{2, A-\mu}\right\| .
$$

Without loss of generality we may assume that $\mu=0$, and then $\left\|L_{2, A}-R_{2, A}\right\|=$ $\left\|L_{2, A}\right\|+\left\|R_{2, A}\right\|$. By Theorem 2.1, this is equivalent to

$$
\left\|L_{2, A}\right\|\left\|R_{2, A}\right\| \in \overline{W\left(-L_{2, A^{*}} R_{2, A}\right)} .
$$

As remarked in the introduction this implies that $\left\|L_{2, A}\right\|\left\|R_{2, A}\right\| \in \sigma\left(-L_{2, A^{*}} R_{2, A}\right)$. But $\sigma\left(-L_{2, A^{*}} R_{2, A}\right)=-\sigma\left(A^{*}\right) \sigma(A)$ (see [2]) and $\|A\|^{2}=\left\|L_{2, A}\right\|\left\|R_{2, A}\right\|$ (see [4]). So there exist $\alpha, \beta \in \sigma(A)$ such that $\|A\|^{2}=-\bar{\alpha} \beta$ ( $\bar{\alpha}$ : complex conjugate of $\alpha$ ). Since $|\alpha| \leq\|A\|$ and $|\beta| \leq \| A \mid$, one can find $\theta \in \mathbb{R}$ such that $\alpha=\|A\| e^{i \theta}$ and $\beta=-\|A\| e^{i \theta}$. So

$$
r\left(\delta_{2, A}\right)=\operatorname{diam}(\sigma(A)) \geq|\alpha-\beta|=2\|A\| .
$$

From the inequality $r\left(\delta_{2, A}\right) \leq\left\|\delta_{2, A}\right\| \leq\left\|\delta_{A}\right\| \leq 2\|A\|$, it follows that

$$
r\left(\delta_{2, A}\right)=\left\|\delta_{2, A}\right\|=\left\|\delta_{A}\right\| .
$$

The reverse implication is obvious since $r\left(\delta_{2, A, B}\right) \leq\left\|\delta_{2, A, B}\right\| \leq\left\|\delta_{A, B}\right\|$.

The next corollary characterizes the $S$-universality of an operator on $H$.

Corollary 3.3. For $A \in \mathcal{L}(\mathcal{H})$, the following are equivalent:

1. A is $S$-universal.

2. $\operatorname{diam}(W(A))=2 \inf _{\lambda \in \mathbb{C}}\|A-\lambda\|$.

3. $\operatorname{diam}(\sigma(A))=2 \inf _{\lambda \in \mathbb{C}}\|A-\lambda\|$.

Proof. From [11] and the inequality of [3],

$$
\operatorname{diam}(\sigma(A)) \leq \operatorname{diam}(W(A)) \leq\left\|\delta_{J, A}\right\| \leq\left\|\delta_{A}\right\|,
$$

it follows immediately that 3$) \Leftrightarrow 2) \Leftrightarrow 1)$. Since $\sigma\left(\delta_{2, A}\right)=\sigma(A)-\sigma(A), r\left(\delta_{2, A}\right)=$ $\operatorname{diam}(\sigma(A))$, so 1$) \Leftrightarrow 3$ ) follows directly from Theorem 3.2.

The following corollary answers negatively questions $2.18,2.19$ and 2.20 of [4].

Corollary 3.4. Every non-zero quasi-nilpotent operator on $H$ is not $S$-universal.

For $A \in \mathcal{L}(\mathcal{H})$, denote by $R(A)$ the radius of the smallest disk containing $\sigma(A)$. In [3], L. Fialkow proved that a subnormal operator is $S$-universal if and only if the diameter of the spectrum is equal to twice the radius of the smallest disk containing it, and asked whether the same holds for an hyponormal operator. The following corollary answers Fialkow's question in the affirmative.

Corollary 3.5. An operator hyponormal $A \in \mathcal{L}(\mathcal{H})$ is $S$-universal if and only if $\operatorname{diam}(\sigma(A))=2 R(A)$.

Proof. This follows from the relation $R(A)=\inf _{\lambda \in \mathbb{C}}\|A-\lambda\|$ (see [11]).

\section{REFERENCES}

[1] Y. A. Abramovich, C. D. Aliprantis, and O. Burkinshaw, The Daugavet equation in uniformly convex Banach spaces, J. Func. Anal. 97(1991), 215-230. MR 92i:47005

[2] A. Brown and C. Pearcy, Spectra of tensor products of operators, Proc. Amer. Math. Soc, 17(1966), 162-166. MR 32:6218

[3] L. Fialkow, A note on norm ideals and the operator $X \longrightarrow A X-X B$, Israel. J. Math. 32(1979), 331-348. MR 81g:47046 
[4] L. Fialkow, Elementary operators \& Applications, Proceedings of the international workshop, Blaubeuren, 1991, pp. 55-113.

[5] I. C. Goheberg and M. G. Krein, Introduction to the theory of linear nonselfadjoint operators, Trans. Math. Monographs, vol.18, Amer. Math. Soc., Providence, RI, 1969. MR 39:7447

[6] P. R. Halmos, A Hilbert space Problem Book, Van Nostrand, Princeton, 1970.

[7] J. Kyle, Numerical ranges of derivations, Proc. Edinburgh. Math. Soc, 21(1978), 33-39. MR 58:7127

[8] C. L. Lin, The unilateral shift and norm equality for bounded linear operators. Proc. Amer. Math. Soc. 127(1999), 1693-1696. MR 99i:47013

[9] R. Schatten, Norm ideals of completely continuous operators, Springer-Verlag, Berlin, 1960. MR 22:9878

[10] S. Y. Shaw, On numerical ranges of generalized derivations and related properties, J. Aust. Math. Soc. Seri. A 36(1984), 134-142. MR 85e:47001

[11] J. Stampfli, The norm of a derivation, Pac. J. Math., 33(1970), 737-747. MR 42:861

Département de Mathématiques, Faculté des sciences Semlalia B.P: 2390 Marrakech, MAROC

E-mail address: barraa@hotmail.com

Département de Mathématiques, Faculté des sciences Semlalia B.P: 2390 Marrakech, MAROC

E-mail address: boumazgour@hotmail.com 